Reporte de Caso

\title{
Divertículo ventricular en pentalogía de Cantrell: entidades infrecuentes, pero no aisladas
}

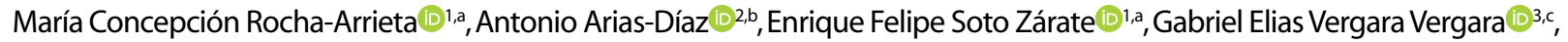 \\ Guillermo José Aristizábal Villa $\mathbb{1}^{4, \mathrm{~d}}$, Gustavo Adolfo Brochet Bayona®4,e
}

Recibido: 22 de octubre de 2021 Aceptado: 05 de diciembre de 2021

Filiación de los autores Universidad de Cartagena. Cartagena, Colombia.

Unidad de Cuidados Intensivos Pediátricos Doña Pilar. Cartagena Colombia.

Centro Diagnóstico de Imágenes y Radiología. Cartagena, Colom-

Hospital Infantil Napoleón Franco Pareja. Cartagena, Colombia

Pediatra.

Cardiólogo pediatra.

Cardiólogo pediatra, imagenología cardiovascular.

Cardiólogo pediatra, hemodinamista.

Cirujano cardiovascular.

\section{*Correspondencia}

María Concepción Rocha- Arrieta

Correo

rochamariac201@gmail.com

Financiamiento

Autofinanciado.

Conflictos de interés

Los autores declaran no tener

conflictos de interés.

Protección de sujetos humanos y animales

Se obtuvo el consentimiento

informado por escrito del paciente para la publicación de este informe

de caso y todas las imágenes

adjuntas.

Confidencialidad de los datos No se revelan datos que identifiquen al paciente.

Citar como:

Rocha- Arrieta MC, Arias-Díaz A Soto Zárate EF, Vergara Vergara GE, Aristizábal Villa GJ, Brochet Bayona GA. Divertículo ventricular en pentalogía de Cantrell: entidades pentalogía de Cantrell: entidade
infrecuentes, pero no aisladas. Arch Peru Cardiol Cir Cardiovasc. 2021;2(4):263-267. doi: 10.47487/ apcyccv.v2i4.162.

\section{RESUMEN}

La pentalogía de Cantrell es una entidad infrecuente caracterizada por una combinación de alteraciones entre las que se destacan: defectos del pericardio, corazón, el diafragma, tercio inferior del esternón y la pared abdominal. Por otro lado, el divertículo cardíaco congénito es una rara malformación cuya presentación se asocia con pentalogía de Cantrell. Presentamos el caso de un escolar con esta afección a quien, durante seguimiento, se le diagnosticó divertículo del ventrículo izquierdo, que precisó manejo quirúrgico y que tuvo evolución favorable. El abordaje de estas condiciones requiere el uso de imágenes cardiovasculares que permiten la toma de decisiones oportunas por parte del equipo tratante.

Palabras clave: Pentalogía de Cantrell; Divertículo; Ectopia cordis; Anomalías cardiovasculares (fuente: DeCS BIREME).

ABSTRACT

\section{Ventricular diverticulum in Cantrell's pentalogy: rare but not isolated entities}

Pentalogy of Cantrell is a rare entity characterized by a combination of alterations, among which the following stand out: defects of the pericardium, heart, diaphragm, lower third of sternum and abdominal wall. On the other hand, congenital cardiac diverticulum is a rare malformation whose presentation is associated with Cantrell's pentalogy in some cases. We present the case of a child with Cantrell's pentalogy who, during follow-up, was diagnosed with left ventricular diverticulum, which required surgical management with a favorable outcome. Addressing these conditions requires the use of cardiovascular images that allow timely decision-making by the treating team.

Keywords: Pentalogy of Cantrell; Diverticulum; Ectopia cordis; Cardiovascular abnormalities (source: MeSH NLM). 


\section{Introducción}

La pentalogía de Cantrell (PC) comprende un conjunto de alteraciones estructurales congénitas de la línea media ventral que incluyen el corazón, pericardio, diafragma, esternón y pared abdominal, que fue descrito por primera vez en $19588^{(1)}$. Las principales manifestaciones son onfalocele supraumbilical, hendidura esternal inferior, defecto en la pared anterior del diafragma, defecto en el pericardio y anomalía intracardiaca (2). Afecta uno de cada 65000 nacidos vivos con predominio del sexo masculino a razón de 2 a $1,2^{(3)}$.

Por su parte, el divertículo ventricular congénito es extremadamente infrecuente, pues representa el 0,05\% entre todas las anomalías cardíacas; se ha documentado aislado o asociado con otra anomalía cardíaca, principalmente con la pentalogía de Cantrell ${ }^{(4)}$.

Presentamos el caso de un escolar al que se le realizó corrección del divertículo cardíaco en contexto de PC, este caso se trae con el fin de destacar las controversias presentes en el manejo de divertículo cardíaco congénito en pacientes asintomáticos; además, destacar la importancia de los métodos diagnósticos de imagen, invasivos como no invasivos en cardiología pediátrica.

\section{Reporte de caso}

Paciente masculino de seis años, con antecedente de diagnóstico prenatal de PC (defecto de la pared anterior tipo hendidura del esternón, hendidura del pericardio, defecto del diafragma con ectopia cardíaca hacia la derecha); inicialmente no se documentó otra anormalidad relacionada al corazón. A los 12 días de vida se le realizó únicamente la corrección del defecto de la pared anterior del tórax en otro centro. Como complicación presentó dehiscencia de sutura lo cual prolongó la hospitalización por tres meses.

El paciente, sin seguimiento médico, consulta a nuestra institución a los 34 meses de vida; asintomático, al examen físico cicatriz quirúrgica subxifoidea, se observa el latido de la punta del corazón por defecto de la caja torácica (Ver video 1, material suplementario), ruidos cardíacos rítmicos, soplo sistólico paraesternal derecho 3/6. En ecocardiograma se observa dilatación leve de cavidades derechas, comunicación interauricular (CIA) de 3,5 mm, insuficiencia tricúspidea leve sin hipertensión pulmonar, función biventricular conservada; con dificultad para determinar el retorno venoso pulmonar y sospecha de comunicación interventricular (CIV) dado la ventana torácica de difícil visualización. No se realizó ecocardiograma transesofágico (ETE) debido a que su realización requiere sedación e intubación orotraqueal en este grupo etario. Se opta por la realización de cateterismo cardíaco como herramienta diagnóstica y terapéutica posible, para defecto del tabique auricular, pero fue diferido por episodios de epistaxis. Luego de tener el aval de otorrinolaringología y hematología pediátrica se realiza el procedimiento a los 54 meses de edad, con los siguientes hallazgos: corazón en mesocardio, CIA tipo ostium secundum $5 \mathrm{x}$ $4,8 \mathrm{~mm}$, dilatación del tronco pulmonar y divertículo ventricular con pedículo de $3 \mathrm{~mm}$ de diámetro que emerge de la pared libre del ventrículo izquierdo, dicho hallazgo no se había detectado en estudios previos.

Con el fin describir mejor los hallazgos y planear la corrección quirúrgica se realiza angiotomografía cardíaca a los 5 años (Figura 1) donde se describe un divertículo del ventrículo izquierdo, que se origina en la pared lateral del tercio medio con las siguientes medidas: boca proximal $3 \mathrm{~mm}$, boca distal $4 \mathrm{~mm}$, longitud $4,3 \mathrm{~cm}$ (de extremo a extremo) y canal $2,5 \mathrm{~cm}$.

Ante los hallazgos de divertículo ventricular, en junta médica se decide dar manejo quirúrgico. Pese a que el menor se encontraba sin datos de compromiso cardiovascular, los padres aceptan el procedimiento ante los eventuales riesgos inherentes al divertículo cardíaco. A los 6 años de edad se realiza procedimiento quirúrgico en el cual - previo a la esternotomía - en la ETE, se encuentra CIA de 3,5 mm y teniendo el dato del QP/QS de 1,3 del cateterismo previo, se consideró que no había indicación quirúrgica de cierre de la CIA. Se procede a realizar exéresis del divertículo, que por la longitud del pedículo y localización no requirió circulación extracorpórea (ver vídeo 2-3, material suplementario), para ello se clampa la base del pedículo comprobándose por ETE que no hay alteraciones de la contractilidad del ventrículo; luego, es resecado y suturado el divertículo al mismo nivel de su origen; se fija el ápex al pericardio con dos puntos sueltos de prolene; la hemostasia se complementa a nivel de zona de disección del divertículo con sellante de fibrina. Además de plastia de pared torácica con los músculos pectorales y reforzamiento de la plastia con malla de polipropileno. Durante el procedimiento el paciente presentó taquicardia ventricular la cual fue manejada con amiodarona. El procedimiento resultó exitoso, con extubación dentro de las primeras $24 \mathrm{~h}$ y buena evolución hasta el momento del egreso. Luego de 6 meses del procedimiento el escolar permanece sin signos de falla cardíaca, en seguimientos periódicos por parte del grupo de cardiología pediátrica institucional.

\section{Discusión}

La pentalogía de Cantrell se ha dividido en varias categorías: completa, parcial e incompleta, cuya diferencia radica en la presencia de todos los defectos o algunos de ellos (cuatro o menos) respectivamente ${ }^{(1)}$. 


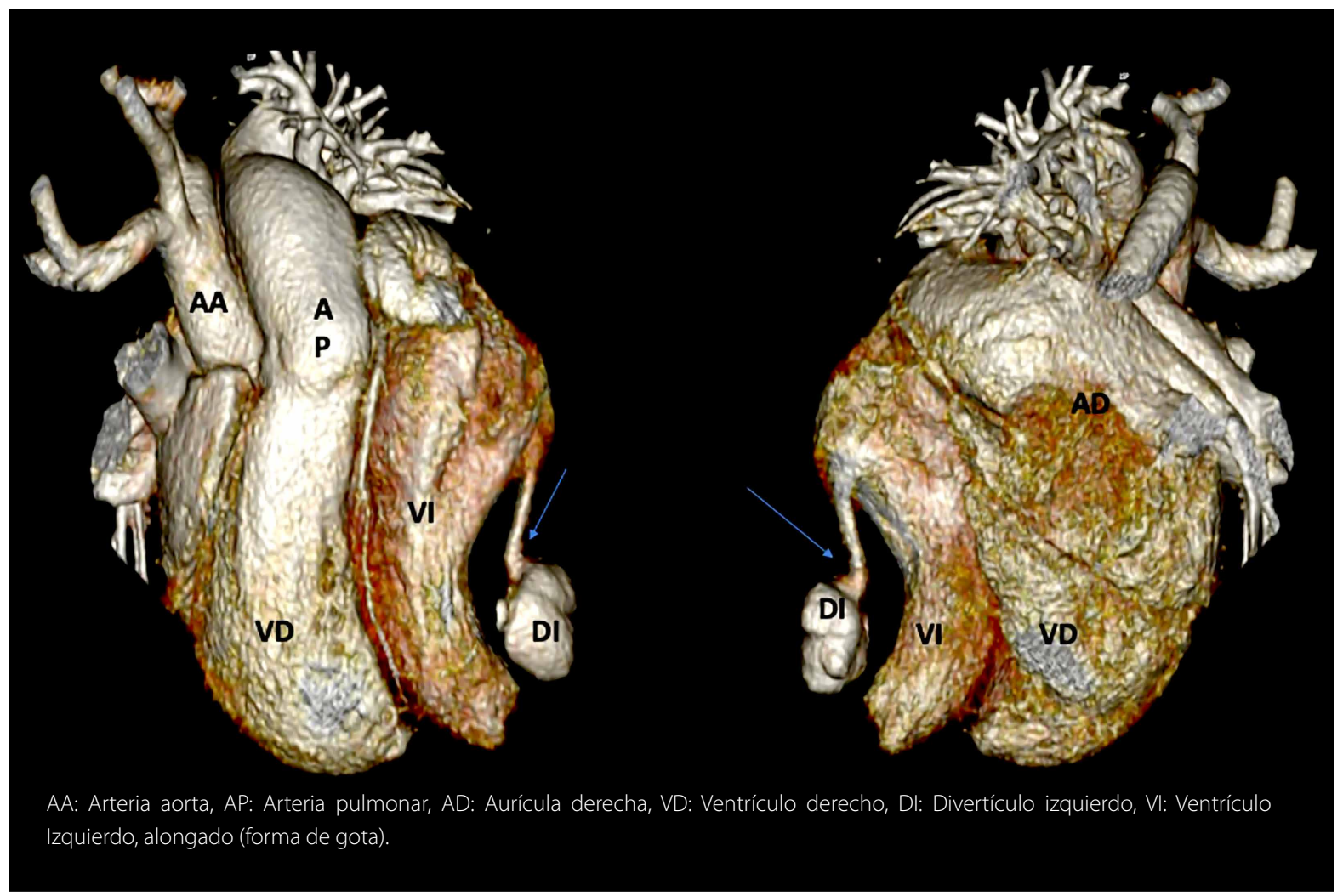

Figura 1. Angiotomografía cardíaca, imagen en reconstrucción 3D. Divertículo del ventrículo izquierdo (DI), que se origina a nivel de tercio medio de la pared lateral del ventrículo izquierdo, con medidas: boca proximal $3 \mathrm{~mm}$, boca distal $4 \mathrm{~mm}$, longitud 4,3 $\mathrm{cm}$ (de extremo a extremo), pedículo $2,5 \mathrm{~cm}$, flecha: pedículo diverticular.

Existen varias teorías con respecto a la fisiopatología de la PC. La teoría inicial sugiere una falla en el desarrollo del mesodermo lateral a las 14 y 18 semanas, generando un fallo de migración y fusión del esternón primordial ${ }^{(5)}$. Por su parte, la teoría más reciente considera que es la ruptura del saco amniótico, con la consiguiente formación de bandas fibróticas que evitan el desarrollo adecuado de los órganos fetales, así como anomalías en el gen 6 y exposición a ciertas drogas. Se describen asociaciones genéticas con la trisomía 13, 18, 21, síndrome de Goltz-Gorlin y el síndrome de Turner ${ }^{(6)}$. También se han asociado a mutaciones genéticas de cromosomas autosómicos y sexuales ${ }^{(7,8)}$ razón por la que se recomienda que los padres deban recibir consejería genética.

Una de las características principales de la PC es la ectopia cordis, subtipo toracoabdominal. Los defectos intracardiacos incluyen CIV (70-100\%), CIA (53\%), tetralogía de Fallot (20\%) y divertículo ventricular izquierdo $(20 \%)^{(9)}$ como se describe en el presente reporte.

El diagnóstico prenatal de PC puede realizarse a través de ecografía de tercera dimensión (3D) o con imágenes más detalladas como la resonancia magnética nuclear fetal. Posnatalmente se requiere radiografía torácica para evaluar la localización del corazón; el ecocardiograma es útil y rápido para evaluar la arquitectura y fisiología intracardiaca; la resonancia magnética (RNM) cardíaca permite determinar defectos pequeños en el diafragma y el pericardio ${ }^{(6)}$.

El manejo de PC requiere estabilización inicial del paciente. La cirugía en sí misma conlleva un riesgo significativo y los pacientes clínicamente estables se tratan inicialmente de forma conservadora. Quirúrgicamente se han optado por métodos correctivos o paliativos estableciendo un abordaje por etapas para disminuir la falla ventilatoria y la mortalidad asociada. La corrección primaria de los defectos cardíacos versus la corrección del defecto diafragmático depende de la presentación clínica del paciente, es decir, de la función cardiopulmonar, la complejidad de malformaciones cardíacas y el tamaño del defecto toracoabdominal ${ }^{(6)}$. Para el cierre a nivel toracoabdominal, se aborda la falta de piel que recubre el corazón y la cavidad abdominal. Luego se procede a cubrir los defectos de la línea media, separar el compartimiento abdominal y pericárdico además de reparar el diafragma. Se utilizan técnicas reconstructivas avanzadas para un cierre adicional, incluido el uso de colgajos y agentes bioprotésicos ${ }^{(1)}$. 
Se estima que la tasa de supervivencia de los pacientes con PC es aproximadamente del $37 \%$ o menos ${ }^{(1)}$. El pronóstico depende de la severidad de la cardiopatía y la extensión de los defectos de la pared abdominal con coexistencia de hipoplasia pulmonar y hernia diafragmática ${ }^{(10)}$. La sepsis, el choque cardiogénico, falla cardiaca y falla multiorgánica se describe como causales de muerte posterior a la cirugía ${ }^{(6)}$.

Con respecto al divertículo, aneurismas y pseudoaneurismas congénitos cardíacos, forman parte de un conjunto de evaginaciones de la pared cardíaca, con una incidencia estimada de 1 en 200000 nacidos vivos; no obstante, la epidemiología actual es difícil de establecer debido a que dicha condición puede pasar inadvertida toda la vida y la mayoría de los datos son obtenidos por reportes de casos o series pequeñas (11); hasta el 2014 la literatura reporta 839 casos $^{(12)}$ en nuestra institución y a nivel local es el primer caso registrado.

Con relación al divertículo congénito del ventrículo izquierdo, es un defecto que se caracteriza por la protrusión sacular de la pared ventricular, conformado por las tres capas del miocardio con un pedículo estrecho, el divertículo se contrae sincrónicamente con el ventrículo izquierdo y puede ocasionar complicaciones como: arritmias ventriculares (11\%), émbolos sistémicos (14\%) y, en el peor de los escenarios, muerte súbita por ruptura ventricular ${ }^{(9,13,14)}$. Dentro de los diagnósticos diferenciales está el aneurisma ventricular congénito que es una protrusión ventricular con base amplia, conformado por tejido fibrótico y miocárdico desorganizado, lo que explica la acinesia o discinesia característica de esta malformación (11). El ecocardiograma podría detectar estos hallazgos; sin embargo, pueden pasar desapercibidos llevando a retrasos en el diagnóstico ${ }^{(14)}$ como en este caso; el cateterismo cardíaco es útil para detectar estas protrusiones, valorar la movilidad cardíaca además de la integridad de las cámaras y arterias coronarias; no obstante, no permite diferenciar entre el divertículo versus aneurisma ventricular. Estas limitaciones se han superado con la angiotomografía y la RNM cardíaca las cuales han tomado relevancia recientemente por ofrecer gran detalle de estructuras cardíacas, extracardíacas, grandes vasos y parénquima pulmonar. En este caso, el diagnóstico se logró con la angiotomografía cardíaca, sin embargo, ante la sospecha de displasia arritmogénica lo más indicado es la RNM que también permite discriminar entre aneurisma y pseudoaneurisma, siendo este último compuesto principalmente de pericardio ${ }^{(9,13)}$. La gran limitante de la RMN es la poca disponibilidad en el país.

En cuanto al manejo de los divertículos congénitos existen controversias. Al ser una entidad poco frecuente, hasta la fecha no hay consenso respecto al tratamiento en pacientes asintomáticos, algunos autores consideran conductas expectantes otros prefieren la resección temprana para evitar complicaciones secundarias a eventos tromboembólicos, ruptura, taquiarritmias o muerte súbita, esta última se presenta principalmente entre los 9 a 15 años para aneurisma y divertículo cardíaco respectivamente ${ }^{(15,16)}$; es por dicha razón que en nuestra institución se prefiere el manejo quirúrgico precoz. Otro aspecto que hay que destacar está relacionado con la técnica quirúrgica, la cual está condicionada al tamaño del defecto y localización; usualmente requiere bomba extracorpórea, aunque un $30 \%$ de los divertículos pueden ser resecados sin el uso de esta herramienta, como en este paciente ${ }^{(15)}$.

Respecto a los riesgos perioperatorios, se ha documentado una mortalidad del 7\% para divertículo cardíaco; en cuanto a la evolución posquirúrgica, generalmente es buena, hay reportes que informan de pacientes seguidos hasta 10 años luego del procedimiento, que se encontraban libres de síntomas ${ }^{(17)}$.

En cuanto a la evolución natural de esta entidad, los datos actuales no son concluyentes lo que hace necesario futuras investigaciones prospectivas ${ }^{(15)}$

En conclusión, la pentalogía de Cantrell constituye un desafío debido a los diferentes tipos de defectos que se pueden presentar como el divertículo ventricular; por esto, el enfoque debe ser integral y personalizado, con un equipo interdisciplinar a lo largo de la vida del paciente; así mismo, utilizar las técnicas de imágenes cardiovasculares que permitan un diagnóstico y manejo oportuno e individualizado.

\section{Referencias bibliográficas}

1. Williams AP, Marayati R, Beierle EA. Pentalogy of Cantrell. Semin Pediatr Surg. 2019. 28(2):106-110. doi: 10.1053/j.sempedsurg.2019.04.006.

2. Duan QJ, Gao Z, Zhang ZW, Li JH, Ma LL, Ying LY. Correct definition of pentalogy of Cantrell. J Perinat Med. 2009;37(4):426. doi: 10.1515/ JPM.2009.065

3. Reyes Caldelas MA, de la Mora Cervantes R, Vargas Hernández SI. "Cantrell's pentalogy"- Angiographic CT evaluation. J Cardiovasc Comput Tomogr. 2020;14(5):e42-e43. doi: 10.1016/j.jcct.2019.01.003.
4. Yang H, Zhu Q, Chen J, Guo N. Congenital left ventricular diverticulum diagnosed by echocardiography. Pediatr Cardiol. 2012;33(4):646-8. doi: 10.1007/s00246-012-0153-7.

5. Correa-Rivas MS, Matos-Llovet I, García-Fragoso L. Pentalogy of Cantrell: a case report with pathologic findings. Pediatr Dev Pathol. 2004;7(6):649-52. doi: 10.1007/s10024-004-9104-5.

6. Jnah AJ, Newberry DM, England A. Pentalogy of Cantrell: Case Report With Review of the Literature. Adv Neonatal Care. 2015;15(4):261-8. doi: 10.1097/ANC.0000000000000209. 
7. Steiner MB, Vengoechea J, Collins RT 2nd. Duplication of the ALDH1A2 gene in association with pentalogy of Cantrell: a case report. J Med Case Rep. 2013;7:287. doi: 10.1186/1752-1947-7-287.

8. Liu Y, Yang J, Jin Z, Zhu H, Yu S, Sun G. One-stage surgical correction in pentalogy of Cantrell with left ventricular diverticulum. J Card Surg. 2014;29(3):413-6. doi: 10.1111/jocs. 12309.

9. Romagnoli A, Ricci A, Morosetti D, Fusco A, Citraro D, Simonetti G. Congenital left ventricular diverticulum: Multimodality imaging evaluation and literature review. J Saudi Heart Assoc. 2015;27(1):61-7. doi: 10.1016/j.jsha.2014.07.004.

10. Asai H, Shingu Y, Ito N, Tachibana T. Norwood Operation of a Neonate With Pentalogy of Cantrell. Ann Thorac Surg. 2020;109(2):e135-e136. doi: 10.1016/j.athoracsur.2019.04.110.

11. Shuplock JM, Kavanaugh-McHugh A, Parra D. Prenatally Diagnosed Congenital Ventricular Outpouchings: An Institutional Experience and Review of the Literature. Pediatr Cardiol. 2020;41(2):272-281. doi: 10.1007/s00246-019-02252-7.

12. Malakan Rad E, Awad S, Hijazi ZM. Congenital left ventricular outpouchings: a systematic review of 839 cases and introduction of a novel classification after two centuries. Congenit Heart Dis. 2014;9(6):498-511. doi: 10.1111/chd.12214.

13. Erol C, Koplay M, Olcay A, Kivrak AS, Ozbek S, Seker M, et al. Congenital left ventricular wall abnormalities in adults detected by gated cardiac multidetector computed tomography: clefts, aneurysms, diverticula and terminology problems. Eur J Radiol. 2012;81(11):3276-81.doi: 10.1016/j.ejrad.2012.03.025.

14. Halpern L, Garabedian C, Worrall NK. Congenital Ventricular Diverticulum or Aneurysm: A Difficult Diagnosis to Make. Case Rep Cardiol. 2018;2018:5839432. doi: 10.1155/2018/5839432.

15. Ohlow MA. Congenital left ventricular aneurysms and diverticula: an entity in search of an identity. J Geriatr Cardiol. 2017;14(12):750-762. doi: 10.11909/j.issn.1671-5411.2017.12.005.

16. Yao M, Wang R, Ye W, Ren C. Surgical treatment of congenital left ventricular diverticulum. J Thorac Dis. 2021;13(1):291-298. doi: 10.21037/jtd-20-3510.

17. Fang $Y, A n Q, Y u T$, Luo S. Partial resection of large congenital left ventricular diverticulum in an infant: a case report. BMC Surg. 2020;20(1):146. doi: 10.1186/s12893-020-00808-6. 